\title{
COPD Exacerbations: A Patient and Physician's Perspective
}

John Linnell $\cdot$ John R. Hurst

Received: October 3, 2019 / Published online: November 8, 2019

(C) The Author(s) 2019

\section{ABSTRACT}

This article, co-authored by a patient affected by chronic obstructive pulmonary disease (COPD) and a respiratory specialist, discusses the patient's experience of living with the disease and, in particular, the impact of COPD exacerbations on his life. The physician discusses the clinical approach to COPD exacerbations. Together, they provide a call to action to improve the management of COPD exacerbations.

Funding AstraZeneca.

Keywords: COPD; Diagnosis; Exacerbation; Management; Quality of life; Symptoms; Screening

Enhanced digital features To view enhanced digital features for this article go to https://doi.org/10.6084/ m9.figshare.10028948.

\section{J. Linnell}

Viroqua, WI, USA

J. R. Hurst ( $\square)$

UCL Respiratory, University College London,

London, UK

e-mail: j.hurst@ucl.ac.uk

\section{Key Summary Points}

This article was co-authored by a patient living with COPD and a respiratory specialist

The patient's experience of COPD, especially the impact of COPD exacerbations, and the clinical approach to COPD exacerbations are discussed

Together, the patient and physician provide a call to action to improve the management of COPD including earlier diagnosis of COPD, a better understanding of COPD exacerbations, a focus on the prevention of COPD exacerbations, and the need for patients and physicians to be partners in care

\section{PATIENT'S EXPERIENCE}

I am John Linnell, 62, and I was diagnosed with chronic obstructive pulmonary disease (COPD) in late 2005 at the age of 49 . Upon my diagnosis, I received routine and normal healthcare for COPD. I was prescribed the typical longacting inhalers and had spirometry done at each visit to my pulmonary doctor. As my COPD 
progressed, I finally had to leave the workforce in late 2011, and I applied for disability via the Social Security Administration. At that time, I happened to know someone with COPD, so I did have a vague understanding of what it was, yet thankfully, my pulmonologist did explain it to me as a term related to emphysema and chronic bronchitis, giving me an overview of each.

Initially, in spite of being college educated and most likely knowing better, I didn't take my COPD too seriously, as it didn't really have much impact on my life, other than causing me to begin a gradual decline of moving more slowly. Living in a large metropolitan area, I had easy access to a great pulmonologist and a wonderful primary care physician (PCP). Nonetheless, as COPD does, it continued to progress. My life had been one of physical activity at work, being involved in marketing promotions at major concerts, golf tournaments, new car expos, etc., which was fun but a lot of walking, moving around, and physical work, which led eventually to my needing to accept that I could no longer be effective in the workplace and I had to leave my career.

Having COPD changed my life significantly. The major change was having to give up my career. My life had suddenly become quite different from that of holding a middle-management position overseeing staff. It seemed I had to reinvent myself, both emotionally and financially, and decide both what to do and how to do it. For myself, I had already decided to 'own' my COPD and learn as much as I could and be a part of my own treatment. I quickly took the position that, rather than COPD being my chronic disease that would progress without a cure, I would consider it 'my condition' that would allow me to do what I wanted, but at a somewhat slower pace. A positive mental attitude became my mantra. I firmly believe to this day that this attitude helps my physical life, as well as affecting my emotional well-being, which I am also convinced lowers my incidence and severity of exacerbations. In spite of having lived with COPD for almost 14 years, and having been stage 4 for the last several, I consider myself fortunate that, until last December, I had never been hospitalized with a COPD exacerbation!

Exacerbation is at first a seemingly complicated word for a patient, which physicians should realize. I consider an exacerbation a sudden worsening of my respiratory symptoms that requires medical intervention, whether it be prescribed oral steroids, and/or antibiotics, and/or being seen by a physician in Urgent Care or an Emergency Room (ER).

Exacerbations, for myself, do not have rhyme or reason. While I have not had an overabundance of them (although even one is more than anyone would want), they do not always manifest themselves, or come on, or develop in the same way. I have had, in my estimation, one or two exacerbations per year for the last 4 years. Prior to that, I have no recollection of needing medical intervention for my COPD.

When I begin to feel a little 'off,' I am aware that something within my respiratory system is not up to par. It can start by my feeling more tired than usual, or perhaps just stopping a bit more often during the day to rest. I find during these times that I utilize the 'pursed lip' breathing technique more often with great success. Pursed lip breathing is a simple technique of inhaling through the nose and taking about twice as long to exhale through the mouth, by pursing your lips, as if blowing. It is fondly referred to as 'smell the roses, blow out the candles'! If these feelings only last a day or so, I chalk it up to just being an 'off' day, which I am sure most people have from time to time. If the intensity lingers and I feel a tightness in my chest, some rib soreness, more fatigue than even an 'off' day might bring, then I decide I need to be proactive by contacting my PCP before I have a more severe exacerbation that could entail a trip to the ER and/or hospitalization.

Fortunately, my healthcare system has an online portal to make appointments or to ask questions of my providers. In my case, my pulmonologist is 45 miles away, so I will usually send an online message to my PCP, who is local, and copy the pulmonologist. In my message, I describe my symptoms such as lethargy, fatigue, shortness of breath, difficulty in accomplishing routine tasks around the house, and tightness of 
the chest. I will ask if he thinks a course of antibiotics and prednisone might be in order, or if he feels I should be seen. Since he has a good understanding of my awareness of my COPD, he will usually agree with a short course of medication (a 5-day course of no taper oral steroids and an antibiotic) and reply that he has sent prescriptions to my local pharmacy for pick-up.

If there is such a thing as my having a typical exacerbation, mine starts with my simply feeling lethargic. I am not sleepy, I just do not have the energy to do much of anything and my pulse oximeter shows that I desaturate rather quickly just walking from one room to the other. I typically do not need supplemental oxygen other than nocturnal use and during exertion. At the outset of an exacerbation, I realize I sometimes need to use supplemental oxygen when doing routine tasks around the house, i.e., going to get the mail, preparing a meal, or unloading the dishwasher.

Last December, I felt an exacerbation coming on with all of the symptoms I have so far described, yet with a more intense lethargy accompanied by fatigue and almost a complete lack of motivation. My oxygen levels were falling to the low 80s just walking across the room to the bathroom. My chest also had a dull ache and I just did not feel well at all. I messaged my physician in the normal manner by using the online portal. Within half an hour, his nurse actually phoned me and said that the doctor felt that, based on how I 'described how I presented,' I would be best served by going to the ER right away, as he felt I needed a more thorough assessment than he could give in an office setting. I went right in and was diagnosed with pneumonia and hospitalized for 3 days. Kudos to my family physician for picking up on it simply based on our working as partners and knowing I seemed to be in more distress than previous times.

Exacerbations for me entail much more than just not feeling well for a few days and taking some additional medications. Even small exacerbations result in less activity, more rest, and somewhat of a road to recovery and time to rebound to what my current COPD baseline is.
It actually takes at least a few weeks to get back to 'my normal.' A severe exacerbation, such as my bout with pneumonia, took much longer to recover from. My supplemental oxygen needs increased significantly, to where my portable oxygen concentrator could not meet my demands, and I needed to utilize oxygen tanks. Rather than needing 2 or $3 \mathrm{lpm}$ for exertion, I needed 3 and sometimes $4 \mathrm{lpm}$ for exertion such as navigating stairs or even walking more than just across the room. I truly thought I had hit a new normal and was not going to completely restore myself to how I was prior to the hospitalization.

It became apparent to me just how quickly I had de-conditioned and lost much of my core strength. As I began slowly exercising, I was able to rebuild, but it was a process that took time and patience. It showed me that staying active, adhering to my long-acting inhaler schedule, and watching for signs of an exacerbation developing are all important in maintaining my current baseline and helping to slow the progression of my COPD.

It made me realize just how fortunate I was to not have had previous exacerbations of that severity. I finally did return to my somewhat 'old normal' after a few months (rather than weeks), yet I still have a 'new normal' and realize that my life can be just as fulfilling, but at somewhat of a slower pace, than it was before the pneumonia. I may die 'with' COPD, but through personal diligence and considering my healthcare professionals as my 'partners in care,' I certainly do not have to die 'from' COPD.

\section{PHYSICIAN'S PERSPECTIVE}

John eloquently describes the two main features of COPD. First, a progressive decline in functional ability as symptoms such as breathlessness worsen with disease progression. Second, and superimposed on this, 'exacerbations': the unpredictable occurrence of periods in which symptoms worsen [1]. John has clearly set out to master his COPD and I would encourage everyone living with COPD to do the samelive life to the full, despite your COPD, and 
know what good care should look like (and ask for this if it doesn't happen).

From a medical perspective, COPD is a complex interplay of an individual's predisposition (e.g., genetic, abnormal lung development) and being exposed to sufficient environmental stimulus (usually tobacco smoke in high-income countries) [1]. COPD is a very common problem, and the third most common cause of death in the world [2]. The term 'COPD' is itself troublesome. COPD is related to, but not the same as, some older terms including chronic bronchitis (cough productive of sputum) and emphysema (loss of alveoli) [1]. The main problem in COPD lies in the smallest airways. COPD is often confused with, and indeed can sometimes overlap with, asthma (though this is usually allergy-driven) [1]. Asthma is also very common, and trying to work out whether someone has asthma or COPD is important, but not always easy.

Too often the diagnosis of COPD is made late, when there is a significant amount of damage in the lung $[3,4]$. The diagnosis can only be made using spirometry [1]. Symptoms such as breathlessness only usually occur when there has already been significant loss of lung function, so clinicians need to get better at thinking about COPD in those at higher risk, such as people with chronic bronchitis or winter chest infections, especially if they have smoked. Spirometry should be repeated regularly to assess the progression of COPD, as John describes, and for people with milder COPD who are not experiencing exacerbations, an annual review may be all that is required to check on progress and to make sure people are receiving the right treatments [1]. Clinicians should be following recognized guidelines to make sure that the care they are delivering is up to date.

John is fortunate, and perhaps a little unusual, in having such ready access to a specialist clinician when he is unwell. The unpredictability of exacerbations makes support at this time really important, and the episode that happened to John last December emphasizes why-this was pneumonia and not a standard exacerbation. It can be difficult for people living with COPD to tell the start of an exacerbation from a 'bad day' and difficult for clinicians to tell a COPD exacerbation from other potential causes of symptom changes in someone with COPD. There is no diagnostic test for an exacerbation, and, at least for now, there is no test that can rapidly and reliably tell whether an exacerbation is due to a virus or a bacteria and so predict the need for treatment, such as antibiotics. Being alert that this episode is somehow 'different' can be very important-to both patient and clinician. I would recommend everyone with COPD make a plan of what to do if an exacerbation were to come along. Who would you contact for advice? How would you reach them? Have you got all the medications you might need in the house? And enough food to last a few days in case you can't get out? If this exacerbation feels in any way different to previous ones, tell your clinician.

We now have many strategies to reduce the risk of exacerbations in people living with COPD. Many of these reduce the level of symptoms too. Some people with COPD appear more likely to get exacerbations than others, which means that treatments can be directed to those who need them most, and 'at risk' patients can be readily identified in the clinic by asking about previous exacerbations [5]. COPD care should first and foremost be about getting the basics right, and that starts with helping someone to quit smoking, if they still smoke. Globally, the risk of developing COPD is further increased by indoor air pollution, such as cooking over open fires in enclosed spaces, and reducing exposures in this situation is much more difficult. People with COPD should be offered vaccinations against influenza (annually) and Pneumococcus [1]. The activity and education class called 'Pulmonary Rehabilitation' is also a very effective way to reduce symptoms, improve exercise performance, and reduce exacerbations, but it still isn't readily available to everyone living with COPD. Both the education and the activity component are important-people learn more about their condition as they get fitter-and I cannot emphasize enough the value and benefit of pulmonary rehabilitation.

When it comes to medicines, clinicians need to think about how best to use these to 
maximize benefit and minimize side effects. For most people with significant COPD, like John, the main medicines used are long-acting bronchodilators, and there are two types of these [long-acting muscarinic antagonists and longacting $\beta_{2}$-agonists]. Inhaled corticosteroids are also commonly used, and, increasingly, clinicians are getting smarter at using inhaled corticosteroids by looking for features that predict who will and who will not benefit [1]. These include features of asthma or a relative elevation in eosinophils. This is helpful because of the side effects that can be associated with inhaled corticosteroids: skin thinning and bruising are the most common, but there can be more serious effects too, such as an increased risk of pneumonia [1]. As technology has progressed, it has become possible to have two and, more recently, all three types of medicine in a single inhaler, which is generally more convenient. It is imperative that people living with COPD know how to use their inhalers correctly [6]. Different types of inhalers need different techniques: you need the right technique for the right inhaler, and the wrong technique may have the same effect as not taking the drug at all. Where these three inhaled medicines are ineffective, there is a range of other more specialist options that can help to reduce symptoms and exacerbations [1]. That said, even with the best care, and despite new drugs on the horizon, exacerbations can still happen from time to time-another reason to make a plan for what to do if one were to occur.

COPD is more common in older people and in people who have smoked, so people living with COPD often have comorbidities [7]. This means that clinicians need to be holistic-not just focusing on the COPD, but on the person as a whole. A good example of this is cardiovascular disease. We know that people living with COPD are at higher risk of heart attacks and strokes than the general population, so making sure that the risk factors, such as high blood pressure and elevated cholesterol, are properly managed is really important [8].

COPD is a major global problem [9]. If we are really going to change future respiratory health, we need to think about exposure reduction, strategies to diagnose the condition earlier, and new treatments that modify disease progression. For the moment, though, by working in partnership with expert patients such as John, we can aim to reduce the burden of COPD for the millions of people living with this condition around the world today.

\section{PATIENT AND PHYSICIAN CALL TO ACTION FOR COPD}

\section{An Earlier Diagnosis of COPD is Needed}

Timely, accurate diagnosis provides the opportunity to protect against COPD progression. Early manifestations of COPD should be followed up with case finding using qualityassured spirometry, e.g., patients presenting with a winter infection and a characteristic profile for COPD should be followed up after resolution of the acute infection. At diagnosis, the clinician should explain in lay terms what COPD means, as initials and acronyms may be frightening and alarming to a patient receiving a potentially life-changing diagnosis.

\section{A Better Understanding of COPD Exacerbations Is Needed by People with COPD and Healthcare Professionals}

A person with COPD and their clinician (e.g., physician, nurse, physiotherapist) may have a different understanding of what a COPD exacerbation is, and often an exacerbation is not accurately reported. Both patients and clinicians may have trouble recognizing the onset of an exacerbation and distinguishing this from symptom variation-a 'bad day.' A COPD exacerbation leaves damage after each episode, and therefore people living with COPD must be encouraged to know how to recognize the symptoms of their COPD exacerbations as soon as they start. The patient and clinician should develop a personalized action plan to help the patient know how to recognize the onset of an exacerbation and understand what proactive steps they need to take to gain control of the episode in order to mitigate the consequences of an exacerbation. 


\section{Focus on the Prevention of Exacerbations}

Given the range of therapeutic interventions available, careful selection of the right approach for managing COPD at the right time is keyprecision medicine. The best way for a clinician to do that is usually by considering the latest guidelines. Education and physical activity, including a discussion of whether pulmonary rehabilitation is appropriate, must be included in the management plan, alongside vaccinations and medication.

\section{Patients and Physicians Need to be Partners in Care}

A better dialogue between a person with COPD and the healthcare professionals surrounding their care is warranted, such that a close therapeutic relationship develops and evolves as the patient's COPD evolves. A close collaboration between the person with COPD and healthcare professionals should be encouraged, such that the patient feels that the clinician is able to take a holistic approach to the management of the patient by considering all aspects of the individual, including other medical and personal difficulties that they may be living with, including their mental well-being. This may also include healthcare professionals being sensitive to language used that could be counter-productive in interpretation by the patient, for example, terms such as 'end stage' should not be used. Patient and carer support groups are valuable in allowing patients the opportunity of sharing experiences with one another and the clinician should play a role in encouraging patients to participate in these support groups.

\section{ACKNOWLEDGEMENTS}

Funding. AstraZeneca funded the journal's Rapid Service Fee. No authors received any compensation in relation to authorship on this manuscript.
Medical Writing and/or Editorial Assistance. Medical writing support, under the direction of the authors, was provided by Caroline Shepherd, BPharm, of CMC Connect, a division of McCann Health Medical Communications Ltd, Macclesfield, UK, which was funded by AstraZeneca, Gaithersburg, MD, USA, in accordance with Good Publication Practice (GPP3) guidelines [10].

Authorship. All named authors meet the International Committee of Medical Journal Editors (ICMJE) criteria for authorship for this manuscript, take responsibility for the integrity of the work as a whole, and have given final approval for the version to be published. The opinions expressed in the manuscript are those of the authors.

Disclosures. J. Linnell reports compensation for services spent in consulting activities from AstraZeneca, Verona Pharma, Mylan/Theravance and from the COPD Foundation PatientPowered Research Network, John Hopkins University, and the University of Illinois at Chicago, in the capacity of presenting the patient perspective within the COPD patient community. J.R. Hurst reports grants, personal fees, and non-financial support from pharmaceutical companies that make medicines to treat COPD.

Compliance with Ethics Guidelines. This article does not contain any new studies with human or animal subjects performed by any of the authors.

Data Availability. Data sharing is not applicable to this article as no datasets were generated or analyzed during the current study.

Open Access. This article is distributed under the terms of the Creative Commons Attribution-NonCommercial 4.0 International License (http://creativecommons.org/licenses/ by-nc/4.0/), which permits any noncommercial use, distribution, and reproduction in any medium, provided you give appropriate credit to the original author(s) and the source, 
provide a link to the Creative Commons license, and indicate if changes were made.

\section{REFERENCES}

1. Global Initiative for Chronic Obstructive Lung Disease. 2019 Report: global strategy for prevention, diagnosis and management of COPD. 2019. http://www.goldcopd.org. Accessed 28 Jun 2019.

2. World Health Organization. Global health estimates 2016: deaths by cause, age, sex, by country and by region 2000-2016. 2018. https://www.who. int/healthinfo/global_burden_disease/estimates/ en/. Accessed 11 Apr 2019.

3. Mapel DW, Dalal AA, Blanchette CM, Petersen H, Ferguson GT. Severity of COPD at initial spirometry-confirmed diagnosis: data from medical charts and administrative claims. Int $\mathrm{J}$ Chron Obstruct Pulmon Dis. 2011;6:573-81.

4. Price D, Freeman D, Cleland J, Kaplan A, Cerasoli F. Earlier diagnosis and earlier treatment of COPD in primary care. Prim Care Respir J. 2011;20:15-22.
5. Hurst JR, Vestbo J, Anzueto A, Locantore N, Mullerova $\mathrm{H}$, Tal-Singer $\mathrm{R}$, et al. Susceptibility to exacerbation in chronic obstructive pulmonary disease. N Engl J Med. 2010;363:1128-38.

6. Usmani OS. Treating the small airways. Respiration. 2012;84:441-53.

7. Bednarek M, Maciejewski J, Wozniak M, Kuca P, Zielinski J. Prevalence, severity and underdiagnosis of COPD in the primary care setting. Thorax. 2008;63:402-7.

8. Smith MC, Wrobel JP. Epidemiology and clinical impact of major comorbidities in patients with COPD. Int $\mathrm{J}$ Chron Obstruct Pulmon Dis. 2014;9:871-88.

9. World Health Organization. Global surveillance, prevention and control of chronic respiratory diseases: a comprehensive approach. 2007. https:// apps.who.int/iris/bitstream/handle/10665/43776/ 9789241563468_eng.pdf. Accessed 30 Jul 2019.

10. Battisti WP, Wager E, Baltzer L, Bridges D, Cairns A, Carswell CI, et al. Good publication practice for communicating company-sponsored medical research: GPP3. Ann Intern Med. 2015;163:461-4. 